\title{
Thermal-Electrical-Mechanical Simulation of High Pressure Spark Plasma Sintering (HP-SPS) Process
}

\author{
Youssef ACHENANI ${ }^{1}$, Abdelkhalek CHEDDADI ${ }^{1, *}$, Malika SAÂDAOUI ${ }^{1}$, Gilbert FANTOZZI \\ ${ }^{1}$ Mohammed V University in Rabat, Mohammadia School of Engineers, Avenue Ibn Sina, P.O. Box 765-Agdal, Rabat, \\ Morocco \\ ${ }^{2}$ Lyon University, National Institute of Applied Sciences of Lyon, MATEIS Laboratory, UMR 5510 CNRS, 20 Avenue \\ Albert Einstein, F-69621 Villeurbanne Cedex, France \\ *Corresponding author: E-mail address: cheddadi@emi.ac.ma
}

\begin{abstract}
In the present work, we investigate the stresses distribution using a high pressure die during SPS (spark plasma sintering) experiments. In this context, we used a finite element modeling (FEM) in the case of the sintering of an alumina sample, chosen as an electrically insulator ceramic material. A thermal sintering cycle is imposed using a control pyrometer of temperature at the $\mathrm{SiC}$ inner die surface.
\end{abstract}

Keywords: Spark Plasma Sintering; Finite element modeling; High pressure; Stress distribution

\section{Introduction}

The Spark Plasma Sintering (SPS) is one of the most attractive consolidation techniques, that combines an application of uniaxial pressure and a very high heating generated by a high current flow. The sintering is achieved at a lower temperature and a shorter period of time compared to more conventional techniques, which allow to enhance the mechanical or physical properties of the materials. This process has become a powerful technology to densify high quality ceramics including nanostructured ceramics [1].

Stresses distribution was studied by Wang et al. [2] in the case of SPS process with classical die, as it was used in previous work [3]. The use of a high pressure die in the SPS configuration allows applying very high pressures compared to conventional hot pressure. Anselmi-Tamburini et al. [4] are the first authors that have developed a high-pressure cell which can use pressures up to $1 \mathrm{GPa}$ for a sample diameter of $5 \mathrm{~mm}$. Recently, in another study published by Sokol et al. [5], the pressure used reaches $1 \mathrm{GPa}$ for a sintering temperature of $1000{ }^{\circ} \mathrm{C}$ for a sample diameter of $8 \mathrm{~mm}$. The same authors [6], applied pressure up to $500 \mathrm{MPa}$ for a sintering temperature varying from $1150{ }^{\circ} \mathrm{C}$ to $1300{ }^{\circ} \mathrm{C}$.
Moreover, the use of the high-pressure cell consisting of a graphite outer die ( $\mathrm{SiC}$ inner die) is mainly used to find the optimal conditions for sintering materials by investigating the key parameters (pressure and sintering temperature), i.e. if the uniaxial pressure is increased, the sintering temperature decreases.

During our HP-SPS experiments, a fracture of the inner die made $\mathrm{SiC}$ is checked. Hence the goal of the employing the FEM modeling to estimate the stress reached in each element of the HP-SPS system.

\section{Finite element modeling}

\subsection{Governing equations}

Taking into account the axial symmetry of the device, the HP-SPS process corresponds to a twodimensional axisymmetric model, limited between the top spacers ends (see Fig. 1). The build model is based on a set of governing equations that involve a three way dynamic coupling of the conservation of the electrical charge, the heat and the mechanical equilibrium equations.

The coupled partial differential equations describing the HP-SPS process, together with the associated initial and boundaries conditions are solved using the COMSOL Multiphysics ${ }^{\circledR}$ finite element package.

The electrical current and temperature variation is governed by the following partial differential equations:

$\vec{\nabla} \cdot \vec{J}=0$

$\rho c_{\mathrm{p}} \frac{\partial \mathrm{T}}{\partial \mathrm{t}}-\nabla \cdot(\lambda \vec{\nabla} \mathrm{T})-\dot{\mathrm{q}}_{\mathrm{J}}=0$

where $\vec{J}=\sigma \vec{E}$ denotes the electrical current density vector, $\sigma$ the electrical conductivity, $\overrightarrow{\mathrm{E}}=-\nabla \mathrm{U}$ the electric field vector, $U$ the electric potential, $\rho$ the density, $c_{p}$ the heat capacity, $T$ the temperature, $t$ the time, $\lambda$ the thermal conductivity and $\dot{\mathrm{q}}_{\mathrm{J}}=\overrightarrow{\mathrm{J}} . \overrightarrow{\mathrm{E}}$ the Joule heating generated by the flowing current per unit volume per unit time. 
The stress distribution is determined by a set of governing equations, including equilibrium and constitutive equations, the stress-strain and the straindisplacements relationship equations:

$-\vec{\nabla} \cdot \sigma_{\mathrm{ij}}=\overrightarrow{\mathrm{F}}$

$\sigma_{\mathrm{ij}}=\mathrm{D} \varepsilon_{\mathrm{ij}}$

$\varepsilon_{\mathrm{ij}}=\frac{1}{2}\left[\nabla \mathrm{u}+(\nabla \mathrm{u})^{\mathrm{T}}\right]$

where $\sigma_{\mathrm{ij}}$ is the stress tensor, $\mathrm{F}$ the applied force, $\mathrm{D}$ the elasticity matrix and $\varepsilon_{\mathrm{ij}}$ the strain tensor.

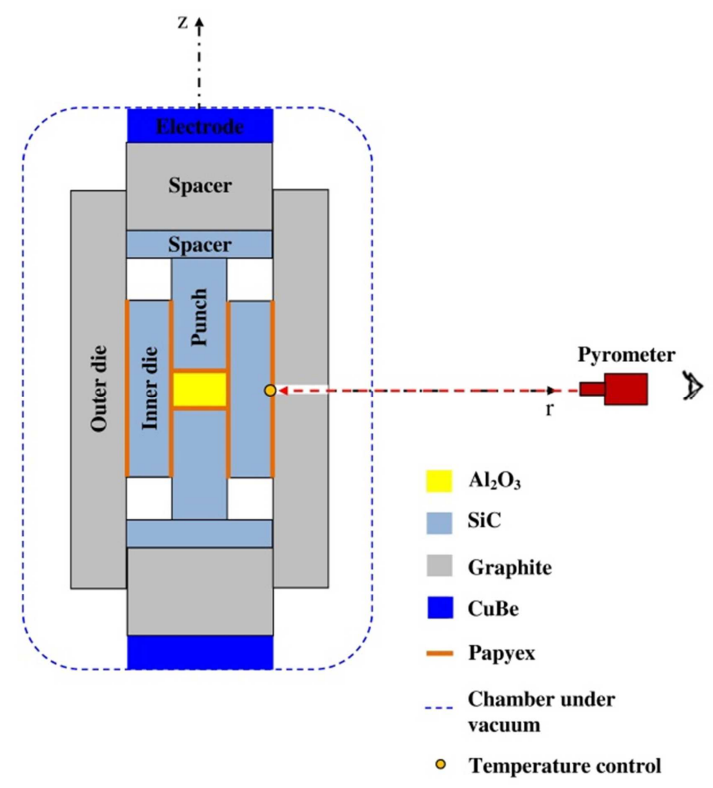

Fig. 1. Schematic view of the HP-SPS system with the temperature control point.

\subsection{Initial and boundary conditions}

An effective electric current $I_{R M S}$ is imposed on the upper surface of the system and the electric potential is zero at its lower surface. To better reproduce the desired thermal sintering cycle $\left(100{ }^{\circ} \mathrm{C} / \mathrm{min}, 1300{ }^{\circ} \mathrm{C}\right.$ maintained for $10 \mathrm{~min}$ ), a PID module reproducing the closed-loop controller configuration has been programmed and integrated into the finite element software. The current intensity is calculated considering the difference, $e(t)$, between prescribed and calculated temperatures at control point (see Fig. 1), as follows:

$$
\mathrm{I}_{\mathrm{RMS}}(\mathrm{t})=\mathrm{K}_{\mathrm{P}} \times \mathrm{e}(\mathrm{t})+\mathrm{K}_{\mathrm{I}} \times \int_{0}^{\mathrm{t}} \mathrm{e}(\tau) \mathrm{d} \tau+\mathrm{K}_{\mathrm{D}} \times \frac{\mathrm{de}(\mathrm{t})}{\mathrm{dt}}
$$

where $K_{P}, K_{I}$ and $K_{D}$ are respectively the proportional, integral and derivative gains, adjusted for an optimum control response.

The initial temperature of the simulations was fixed at 27 ${ }^{\circ} \mathrm{C}$ and two main heat transfer boundary conditions were applied (Fig. 2).
A constant uniaxial force of $78.5 \mathrm{kN}$, applied to the top spacer while the bottom one remains fixed, provides a $1 \mathrm{GPa}$ macroscopic load on the sample (of diameter $\phi=$ $10 \mathrm{~mm}$ and $5 \mathrm{~mm}$ in thickness).
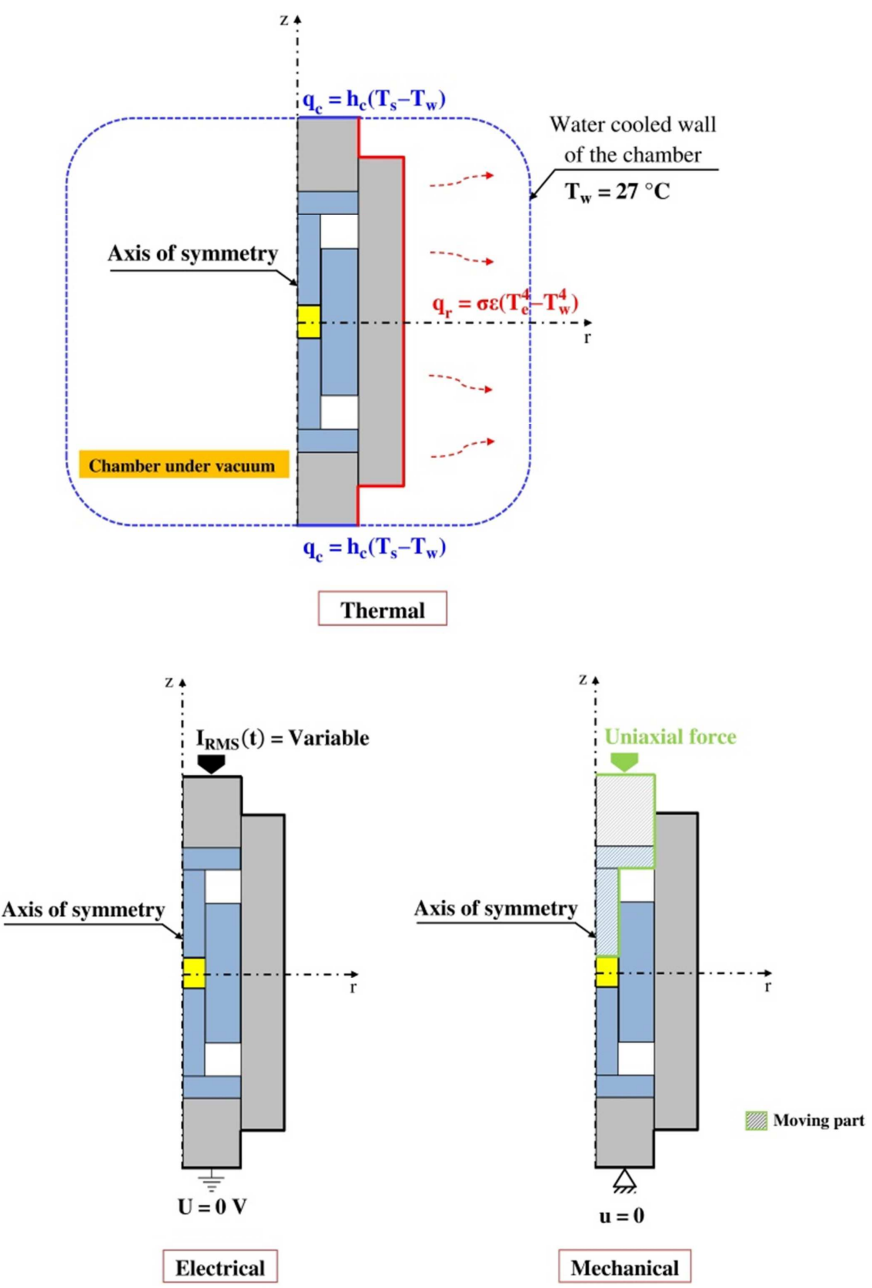

Fig. 2. Thermal, electrical and mechanical boundary conditions applied to the finite element model.

\section{Results and discussion}

Both configurations of the HP-SPS System of different geometric dimensions were studied. Stresses distribution along the radial central line (Or) and the axial central line $(\mathrm{Oz})$ is represented on Fig. 3. All the stresses are calculated at the end of the dwell time. 


\section{Configuration $\mathbf{N}^{\circ} \mathbf{1}$}

SiC outer die: $\phi_{\text {ext }} 30 \times \mathrm{H} 30 \mathrm{~mm}$ Graphite outer die: $\phi_{\text {ext }} 80 \times \mathrm{H} 70 \mathrm{~mm}$

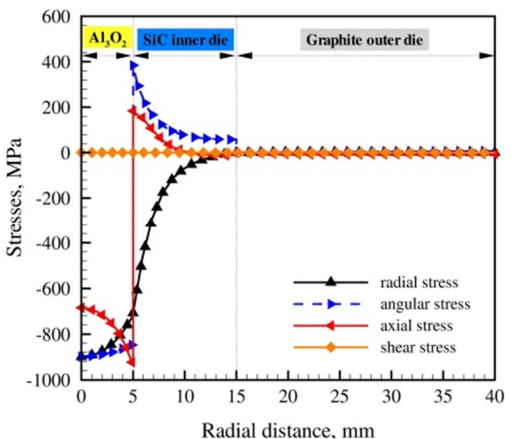

SiC spacer: $\phi 30 \times \mathrm{H} 5 \mathrm{~mm}$ Graphite spacer: $\phi 30 \times \mathrm{H} 30 \mathrm{~mm}$
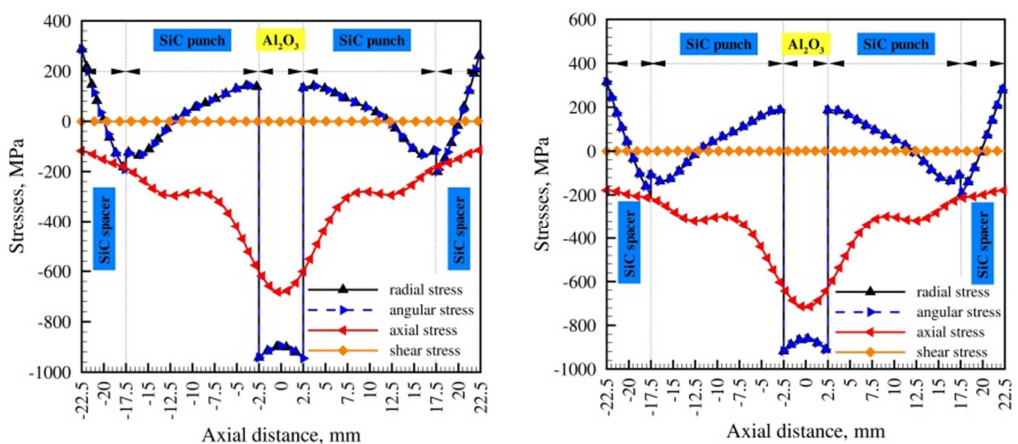

Fig. 3. Comparison of the stress distribution in both configurations 1 and 2 along the cross-section of the middle of sample, inner and outer die (on top) and across the axial center of the apparatus (on bottom).

From Fig. 3, it is important to note that, in the case of the second configuration, in the radial plane, in the $\mathrm{SiC}$ inner die, the angular stress becomes higher (447 $\mathrm{MPa}$ at the contact interface with the sample) compared to that of the first configuration $(387 \mathrm{MPa}$ at the contact interface with the sample). For the radial stresses, an increase was noted of the order of $42 \mathrm{MPa}$ at the contact interface with the sample, passing from $-666 \mathrm{MPa}$ in the case of the second configuration instead of $-708 \mathrm{MPa}$ in the case of the first one. In contrast to the axial stresses that decreased by about $62 \mathrm{MPa}$ at the contact interface with the sample, passing from $121 \mathrm{MPa}$ in the case of the second configuration instead of $183 \mathrm{MPa}$ in the case of the first one.

In the axial plane, the same trend can be observed in terms of the variation of the stresses for the two studied configurations. It has been found that the radial, angular and axial stresses are relatively increased in the case of the second configuration, compared to the first one. Furthermore, it is notable that in the case of the classical die, according to Wolff et al. [7] the largest radial stress is located at mid-height and near the sample. A high stress concentration was found at the contact interface: sample/die, generating compression in the die. As a result, the die is subjected to very high angular tensile stresses. These stresses are recognized as the cause of the fracture of the die [7], as it was found during our HPSPS experiments.

\section{Conclusion}

The study demonstrates the potential of FEM modeling for understanding and analysing the stresses distribution in the whole HP-SPS device.

The main conclusions of the present work are summarized as follows:

-The stresses distribution depends strongly on the dimensions and the properties of the HP-SPS tooling. -Stress gradients have been identified in the sample in the radial and axial directions.

\section{References}

[1] O. Guillon, J.G. Julian, B. Dargatz, T. Kessel, G. Schierning, J. Räthel, M. Herrmann, Fieldassisted sintering technology/spark plasma sintering: Mechanisms, materials, and technology developments, J. Adv. Eng. Mater. 16 [7] (2014) 830-849.

[2] X. Wang, S.R. Casolco, G. Xu, J.E. Garay, Finite element modeling of electric currentactivated sintering: The effect of coupled electrical potential, temperature and stress, Acta Mater. 55 [10] (2007) 3611-3622.

[3] Y. Achenani, M. Saâdaoui, A. Cheddadi, G. Bonnefont, G. Fantozzi, Finite element modeling of spark plasma sintering: Application to the reduction of temperature inhomogeneities, case of alumina, Mater. Des. 116 (2017) 504-514.

[4] U. Anselmi-Tamburini, J.E. Garay, Z.A. Munir, Fast low-temperature consolidation of bulk nanometric ceramic materials, Scripta Mater. 54 (2006) 823-828.

[5] M. Sokol, M. Halabi, S. Kalabukhov, N. Frage, Nano-structured $\mathrm{MgAl}_{2} \mathrm{O}_{4}$ spinel consolidated by high pressure spark plasma sintering (HPSPS), J. Euro. Ceram. Soc. 37 (2017) 755762.

[6] M. Sokol, S. Kalabukhov, M.P. Dariel, N. Frage, High-pressure spark plasma sintering (SPS) of transparent polycrystalline magnesium aluminate spinel (PMAS), J. Euro. Ceram. Soc. 34 (2014) 4305-4310.

[7] C. Wolff, S. Mercier, H. Couque, A. Molinari, F. Bernard, F. Naimi, Thermal-electricalmechanical simulation of the nickel densification by spark plasma sintering. Comparison with experiments, Mech. Mater. 100 (2016) 126-147. 